\title{
AIAA 2000-2360 The Application of the BGK Model in Particle Simulations
}

Michael A. Gallis and John R. Torczynski

Engineering Sciences Center

Sandia National Laboratories

Albuquerque New Mexico 87185-0826

\section{4th AIAA Thermophysics Conference 19-22 June 2000 / Denver, CO}

For permission to copy or republish, contact the American Institute of Aeronautics and Astronautics 1801 Alexander Bell Drive, Suite 500, Reston, VA 20191 


\section{DISCLAIMER}

This report was prepared as an account of work sponsored by an agency of the United States Government. Neither the United States Government nor any agency thereof, nor any of their employees, make any warranty, express or implied, or assumes any legal liability or responsibility for the accuracy, completeness, or usefulness of any information, apparatus, product, or process disclosed, or represents that its use would not infringe privately owned rights. Reference herein to any specific commercial product, process, or service by trade name, trademark, manufacturer, or otherwise does not necessarily constitute or imply its endorsement, recommendation, or favoring by the United States Government or any agency thereof. The views and opinions of authors expressed herein do not necessarily state or reflect those of the United States Government or any agency thereof. 


\section{DISCLAIMER}

Portions of this document may be illegible in electronic image products. Images are produced from the best available original document. 


\title{
The Application of the BGK Model in Particle Simulations
}

\author{
Michael A. Gallis and John R. Torczynski \\ Engineering Sciences Center. \\ Sandia National Laboratories* \\ Albuquerque New Mexico 87185-0826
}

\begin{abstract}
A collision model for the Direct Simulation Monte Carlo (DSMC) method is presented. The collision model is based on the $\mathrm{BGK}^{1}$ equation and makes use of the Cercignani ${ }^{2}$ ellipsoidal distribution to incorporate the effects of heat conductivity. Results obtained by the DSMC method and its BGK and BGKC modifications for a $10^{\circ}$ wedge and a flat plate are presented and discussed.
\end{abstract}

\section{Introduction}

An area of interest for particle simulation codes is that of dense near-equilibrium flows in Micro Electromechanical Systems (MEMS) in which the scales of the phenomena make the application of continuum techniques questionable. However, the extension of particle simulation methods to highdensity flows is usually associated with very small time steps needed to resolve the large number of collisions that take place each time step. The computational load that high-density flows impose on particle simulations is such that it challenges even the most modern computer platforms.

MEMS flows are typically subsonic - the mean velocity is significantly smaller than the thermal velocity of the particles. As a result, a particle may suffer several collisions within a single time step. Simulation of all these collisions is very timeconsuming, and it does not add any information to the flow since these flows are already in near-equilibrium or equilibrium. The same issue appears when simulating charged flows, in which a charged particle may suffer many collisions within a time step.

The issue of modeling collisional processes extends back nearly three decades to the early days of digital computers. One of the early approaches to reduce the computational load was to replace the collision operator of the Boltzmann equation with simpler forms that maintain most of the fundamental properties of the original equation. The idea behind the simplification of the collision term of the Boltzmann equation is that most of the details of the two-body interactions are not important in reproducing some of the experimentally measured quantities.

In this paper extensions of some of these methods, that were initially proposed for the analytic solution of the Boltzmann equation, are investigated as possible alternatives to reduce the computational load associated with modeling high-density flows. The $\mathrm{BGK}^{\mathrm{l}}$ equation (named after its authors Bhatnagar, Gross and Krook) is used as the starting point, and related approaches are developed by adding more sophisticated distributions and increasing its physical accuracy.

\section{The Boltzmann equation}

The velocity distribution function $f$ in the sixdimensional phase space $(\vec{v}, \vec{r})$ provides a complete description of a gas at the molecular level. The relationship between the velocity distribution function and the variables it depends on is given by the Boltzmann equation:

$$
\begin{aligned}
\frac{\partial}{\partial t}(n f) & +\vec{v} \cdot \frac{\partial}{\partial \vec{r}}(n f)+\vec{F} \cdot \frac{\partial}{\partial \vec{v}}=\left[\frac{\partial}{\partial t}(n f)\right]_{\text {collision }} \\
& =\int_{-\infty}^{\infty} \int_{0}^{4 \pi} n^{2}\left(f^{*} f_{1}^{*}-f f_{1}\right) c_{r} \sigma d \Omega d \vec{v}
\end{aligned}
$$

where $f$ and $f_{l}$ are the pre-collision distribution functions of the two colliding particles and $f^{*}$ and $f^{*} t$ are the post-collision distribution functions. In the same equation $n$ is the number density, $\vec{F}$ is an

"Sandia is a multiprogram laboratory operated by the Sandia Corporation, a Lockheed Martin Company, for the United States Department of Energy under Contract DE-AC04-94AL85000. 
external force that applies to the particles, $c_{r}$ is the relative velocity of the colliding particles, $\sigma$ is the cross section of the binary collision, and $\Omega$ is the solid angle. The term on the right-hand side of the Boltzmann equation is the collision term. The details of the binary collision are included in the collision cross section.

\section{The BGK equation}

The complexity of the nonlinear structure of the collision integral of the Boltzmann equation led to the development of simplified models that retain most of the properties of the Boltzmann equation but are not derived directly from it. Bhatnagar, Gross and Krook ${ }^{1}$ (BGK) proposed a simplification that takes the form

$$
\left[\frac{\partial}{\partial t}(n f)\right]_{\text {collision }}=n v\left(f_{o}-f\right)
$$

where $f_{o}$ is the Maxwellian distribution corresponding to the local temperature and average velocity, and $v$ is the collision frequency. Examination of the BGK model indicates that it reproduces the equilibrium solution $f=$ $f_{o}$ at equilibrium. The BGK equation also reproduces the correct moments and satisfies the H-theorem. For the highly collisional regime, and when used with the Chapman-Enskog expansion, it leads to the NavierStokes equations. In this equation the term $n v f_{o}$ represents the collisions replenishing the equilibrium distribution $f_{o}$, and the term $n v f$ represents the collisions depleting molecules out of distribution $f$.

The physical interpretation of the assumption that the replenishing number of collisions is given by $n v f_{o}$ is that post-collision molecules are selected from a Maxwellian distribution at the local average velocity and temperature. It is evident that this behavior can readily be incorporated in a particle code. By doing so, the burden of calculating individual collisions for each particle is avoided.

A difficulty with the BGK model is the definition of the collision frequency, $v$. One way to specify the collision frequency is with the aid of the coefficient of viscosity $\mu$.

$$
\mu=\frac{n k T}{v}
$$

where $T$ is the temperature of the cell and $k$ is the Boltzmann constant.

A possible modification of the BGK model is to allow the collision frequency to depend on the molecular thermal velocity instead of being locally constant. This assumption is supported by experience with most molecular models and becomes important for high molecular thermal speeds. The assumption of a velocity-dependent collision frequency adds to the computational load of the simulation since the calculation of the collision frequency has to be repeated for each particle instead of just once for each cell. For the purposes of this study, the velocity-dependent collision frequency was ignored.

The BGK model has the inherent feature that the Prandtl number is unity for all cases. This is a significant disadvantage for cases in which the thermal conductivity plays an important role. Instead of using the coefficient of viscosity, the collision frequency can be specified using the coefficient of thermal conductivity $K$ :

$$
K=\frac{5}{2}\left(\frac{k}{m}\right) \frac{n k T}{v} .
$$

where $m$ is the mass of a particle.

\section{The ellipsoidal statistical model (ES)}

Despite the shortcomings of the BGK equation, it is able to reproduce the main features of the Boltzmann equation. Several modifications to the BGK equation have been proposed at the expense of the simplicity of the model. Cercignani ${ }^{2}$ proposed a modification to the BGK equation that would allow it to reproduce the same viscosity and thermal conductivity as the full Boltzmann equation. To do this the Prandtl number is introduced as a parameter in the equation. The generalization of the BGK equation is obtained by the replacement of the Maxwellian distribution with a local anisotropic three-dimensional Gaussian, referred to as the ellipsoidal statistical (ES) model:

$$
f_{o}=\rho \pi^{-3 / 2}(\operatorname{det} A)^{1 / 2} \exp \left[-\sum_{i, j=1}^{3} A_{i j} c_{i} c_{j}\right]
$$

where the matrix $A$ is:

$$
A_{i j}=\left[(2 R T / \mathrm{Pr}) \delta_{i j}-2(1-\mathrm{Pr}) p_{i j} /(\rho \mathrm{Pr})\right]^{-1}
$$

where $R$ is the universal gas constant, $\rho$ is the local density of the flow, Pr is the Prandtl number, and $p_{i j}$ is the pressure tensor. The pressure tensor is calculated from the particle velocities according to the following:

$$
p_{i j} / \rho=\int c_{i} c_{j} f d v
$$

A disadvantage of this method is that it is not known if it satisfies the H-theorem. However, it reproduces the correct moments of the Boltzmann equation like the original BGK.

The calculation of matrix $A$ and its inversion can be a time-consuming operation. This operation can be greatly simplified if it is assumed that the offdiagonal terms are smaller that the diagonal terms. In this case the ES model can be expressed as a modification to random vectors chosen from the equilibrium Maxwellian distribution. 
If $\vec{c}^{*}$ is a random vector chosen from a Maxwellian of temperature $T$, the modifier tensor $S$ is applied to the velocity vector according to:

$$
\vec{c}=S \vec{c}^{*}
$$

so that $\vec{c}$ is a velocity vector chosen from the ES distribution. The tensor $S_{i j}$ is given by:

$$
S_{i j}=\left(\frac{2 k T}{m}\right)^{1 / 2}\left[\begin{array}{l}
\delta_{i j}-\frac{1}{2}\left(\frac{1-\mathrm{Pr}}{\mathrm{Pr}}\right) \\
\left\{\frac{m}{k T} \frac{p_{i j}}{\rho}-\delta_{i j}\right\}
\end{array}\right] \text { (9) }
$$

The BGK model with the Cercignani ES model is herein termed BGKC from the initials of the authors.

\section{Application of the method}

The main requirement for a method that performs collisions of particles with a background distribution is local conservation of momentum and energy. Energy and momentum conservation are conserved only in the limit that the number of particles in a cell is very large. However, failure to conserve energy and momentum exactly may introduce a random walk into the calculation. An alternative to using a very large number of particles per cell is to calculate the total momentum and energy in a cell before the collisions and to adjust the velocities of the particles in the cell after the collisions to ensure that momentum and energy are exactly conserved. However, this operation has a computational overhead, so the BGKC method may be slower than the DSMC method in a collisionless or near-collisionless regime, while it is faster when the number of collisions increases.

\section{Computational method}

The Sandia DSMC code Icarus was used for the simulations. Icarus was developed following Bird's approach $^{3}$ by Bartel and co-workers ${ }^{4}$ for massively parallel applications and can be run on a variety of computational platforms. The calculations presented here were performed on 512 nodes of a 1024-node NCUBE parallel computer with a maximum speed of 1 GFLOP. A calculation typically used 350,000 particles and required slightly less than 10 hours of run time to produce statistically acceptable results.

\section{Computational results}

\section{Argon flow over a $10^{\circ}$ wedge}

The first test case examined is that of the flow over a $10^{\circ}$ wedge. The sides of the wedge are assumed to be specularly reflecting. The gas was pure argon at an ambient temperature of $180 \mathrm{~K}$ and a number density of $10^{22} \mathrm{~m}^{-3}$ with an upstream velocity of $1365 \mathrm{~m} / \mathrm{s}$. The domain was discretized with 25,000 cells and a time step of $10^{-8} \mathrm{~s}$ was used. The downstream boundary condition was assumed to be non-reentrant (vacuum), which is acceptable for the supersonic flow downstream of an oblique shock. This test case was simulated with the DSMC, BGK and BGKC methods. Steady-state conditions were observed after 5000 time steps while the code executed a total of 65,000 time steps. To ensure that the results were fully converged and grid-independent, a number of additional runs were made in which the grid density, the number of particles, and the time step were successively modified. In these runs the total number of cells was changed from 25,000 to 6,500 (cells four times as large), the number of particles was halved and the time step was first reduced by one order of magnitude and subsequently doubled. All of these runs gave identical results, indicating that the results presented here were independent of the time step, the cell size, and the number of particles. Caution was also exercised to ensure that all cells were smaller than a third of the local mean free path and the particles were not moving more than a quarter of the local mean free path in every move.

This test case is particularly interesting for validation purposes because both the post-shock conditions and the angle the shock forms with the upstream velocity can be analytically calculated. The shock angle $\beta$ is given by (see Anderson ${ }^{5}$ ):

$$
\tan \theta=\frac{\cot \beta\left(M \sin ^{2} \beta-1\right)}{\frac{\gamma+1}{2} M^{2}-\left(M^{2} \sin ^{2} \beta-1\right)}(10)
$$

where $M$ is the free stream Mach number, $\theta$ is the angle of the wedge ( $10^{\circ}$ in this case), and $\gamma$ is the specific heat ratio. There are two solutions to this equation; the first one representing a weak oblique shock and the second one representing a strong oblique shock. In the above situation, the weak solution applies. For this test case the pre- and postshock conditions are given in Table 1.

Figure 1(a-c) presents the number-density contour lines as calculated by the DSMC, BGK, and BGKC methods. Figures $2(a-c)$ in the same fashion present the corresponding translational temperature contour lines.

A qualitative comparison indicates that all three simulations are in agreement about the basic characteristics of the flow field. In all cases the flow travels from the left to the right. At $x=y=0$, the location of the leading edge of the wedge, the shock layer forms. The flow downstream reaches a maximum number density that is about double the free stream number density. The fact that the DSMC, BGK 
and BGKC solutions predict the same downstream temperature and number density confirms that the new methods do not artificially increase or decrease the momentum and energy content of the flow.

\begin{tabular}{|l|l|}
\hline \multicolumn{2}{|c|}{ Upstream Conditions } \\
\hline Molecular Mass & $39.99 \mathrm{amu}$ \\
\hline Specific Heat Ratio & $5 / 3$ \\
\hline Number Density & $10^{22} \mathrm{~m}^{-3}$ \\
\hline Temperature & $180 \mathrm{~K}$ \\
\hline Speed of Sound & $249.75 \mathrm{~m} / \mathrm{s}$ \\
\hline Free Stream Velocity & $1365 \mathrm{~m} / \mathrm{s}$ \\
\hline Mach Number & 5.465 \\
\hline \multicolumn{2}{|c|}{ Downstream Conditions } \\
\hline Number Density & $2.1 \times 10^{22} \mathrm{~m}^{-3}$ \\
\hline Temperature & $335 \mathrm{~K}$ \\
\hline Speed of Sound & $341 \mathrm{~m} / \mathrm{s}$ \\
\hline Velocity & $1304 \mathrm{~m} / \mathrm{s}$ \\
\hline Mach Number & 3.823 \\
\hline Wedge angle & $10^{\circ}$ \\
\hline Shock angle & $19.55^{\circ}$ \\
\hline
\end{tabular}

Table 1. Shock wave conditions

Comparing the three figures in more detail, the BGKC and DSMC solutions are seen to be in remarkable agreement thoughout the domain. The angle that the temperature and number-density contour lines form with the horizontal is $19-20^{\circ}$, as theoretically predicted.

Figure 2(a-c) presents the temperature contour lines for the DSMC, BGK and BGKC solutions. The shock layer formation is seen to occur at the leading edge of the wedge. The temperature increases from 180 $\mathrm{K}$ in the free stream to $325 \mathrm{~K}$ in the downstream area. The computational domain is not long enough for the flow to reach the equilibrium temperature, $336 \mathrm{~K}$.

Again, the BGKC and the DSMC solutions are in very good agreement throughout the flow field. A minor difference is noted in the location of the $185 \mathrm{~K}$ contour line. The BGKC solution appears to be about $5 \%$ thicker than the DSMC solution, whereas the BGK solution appears to be $5 \%$ thinner than the DSMC solution.

It is of particular interest to observe the $325 \mathrm{~K}$ contour line close to the wedge surface. The BGKC solution is in excellent agreement with the DSMC solution in this region, while the BGK solution differs appreciably from the DSMC solution. This is attributed to the more accurate heat conductivity modeling of the BGKC method in comparison with the BGK.

\section{Argon flow over a flat plate}

The previous example indicated that the BGKC solution was in better agreement with the DSMC solution than the BGK was, especially regarding heat-transfer-related phenomena. To examine this idea, a second series of computational experiments was performed with the same ambient gas flow over a diffusely reflecting flat plate. The flow is again from left to right, and the leading edge at $(0,0)$ is the transition point between specular and diffuse reflection.

The number-density contour lines are shown in Figure 3(a-c) for the DSMC, BGK, and BGKC methods, respectively. The contour lines indicate that the boundary layer and the oblique shock separate about 20 mean free paths downstream of the leading edge. The number density across the shock increases at first but then decreases to a value below the free stream density in the area between the boundary layer and the shock.

As in the previous section, the BGKC solution agrees much more closely with the DSMC solution than the BGK one does. Agreement degrades somewhat in the low-density area behind the shock. The DSMC solution presents a smaller area in the $1.5 \times 10^{22} \mathrm{~m}^{-3}$ number-density contour line than the BGKC. It is interesting to note the almost excellent agreement in the area of the boundary layer. The discrepancy in the $1.5 \times 10^{22} \mathrm{~m}^{-3}$ contour line should be attributed to highly non-equilibrium phenomena involved in the boundary layer-shock interaction. The termination of the boundary layer at $x=0.005 \mathrm{~m}$ should is attributed to the supersonic boundary condition on the exit plane.

The temperature contour lines are presented in Figure 4(a-c). The formation of the boundary layer is associated with the rapid increase of the temperature close to the leading edge, where the temperature increases from $180 \mathrm{~K}$ to $850 \mathrm{~K}$. The temperature of the flow increases both from the free stream and the plate to the core of the flow. The abnormal termination of the temperature contour lines on the upper boundary ( $y$ $=0.003 \mathrm{~m})$ and the right hand side $(x=0.005 \mathrm{~m})$ can be attributed to the boundary conditions: a specular reflecting wall for the upper boundary and vacuum for the right boundary. Qualitative agreement was achieved between all three simulations. Again the DSMC and the BGKC solutions were found to be in good agreement, with differences of less than $10 \%$ throughout the domain with the exception of the precursor shock area. The differences in this region may be related to the non-equilibrium conditions that prevail in the corresponding areas of the flow field.

It should be noted that the conditions of these simulations do not favor the BGKC model since significant non-equilibrium regions are found. The BGKC model can be used with confidence only in areas of near-equilibrium. However, its application to non-equilibrium situations indicates that even in areas 
of non-equilibrium the model works with reasonable accuracy.

\section{Conclusions}

A new approximate collisional model for the Boltzmann equation has been developed. The aim of the new model is to reduce the computational load for flows in near equilibrium. Application of the method in situations of near-equilibrium indicates that excellent agreement can be expected. Areas such as mild shocks and boundary layers can be predicted accurately by the BGKC model. Even in areas with significant nonequilibrium, the new model is in reasonable agreement with the DSMC method.

\section{Acknowledgements}

The authors would like to thank Dr. Wahid L. Hermina, Dr. Matthew M. Hopkins and Dr. Richard J. Buss of Sandia National Laboratories for their helpful comments. This work was performed at Sandia National Laboratories. Sandia is a multiprogram laboratory operated by the Sandia Corporation, a Lockheed Martin Company, for the United States Department of Energy under Contract DE-AC0494AL85000.

\section{References}

${ }^{1}$ Vincenti W.G. and Kruger C.H., Introduction to Physical Gas Dynamics, Krieger Publishing Co, 1986.

${ }^{2}$ Cercignani C., The Boltzmann Equation and its Applications, Springer-Verlag, 1988.

${ }^{3}$ Bird G.A., Molecular Gas Dynamics and the Direct Simulation of Gas Flows, Oxford University Press, 1994.

${ }^{4}$ Bartel T.J., Plimpton S., Johannes J.E., Payne J., Icarus: A 2D Direct Simulation Monte Carlo (DSMC) Code for Parallel Computers, Users Manual 3.2. Sandia Report SAND96-0591, 1996.

${ }^{5}$ Anderson J.D., Modern Compressible Flow, McGrawHill, 1990. 


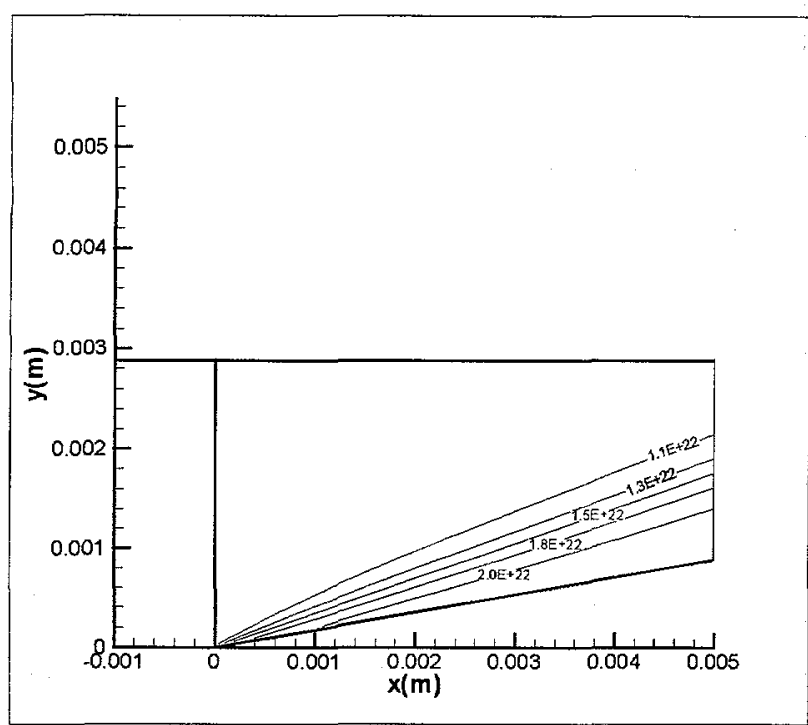

Figure 1a. Flow over a $10^{\circ}$ wedge. DSMC Simulation. Number density $\left(\mathrm{m}^{-3}\right)$

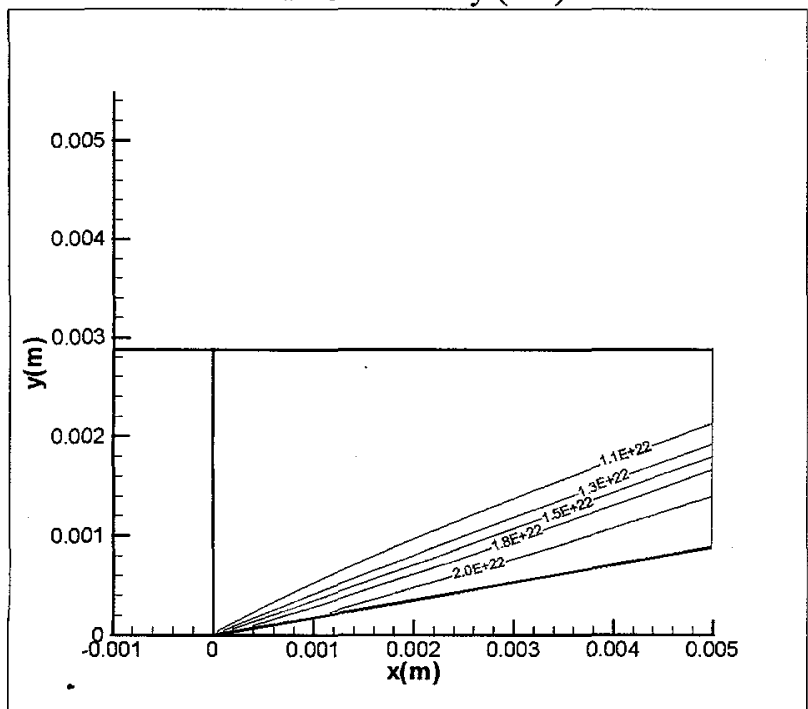

Figure 1b. Flow over a $10^{\circ}$ wedge. BGK Simulation. Number density $\left(\mathrm{m}^{-3}\right)$

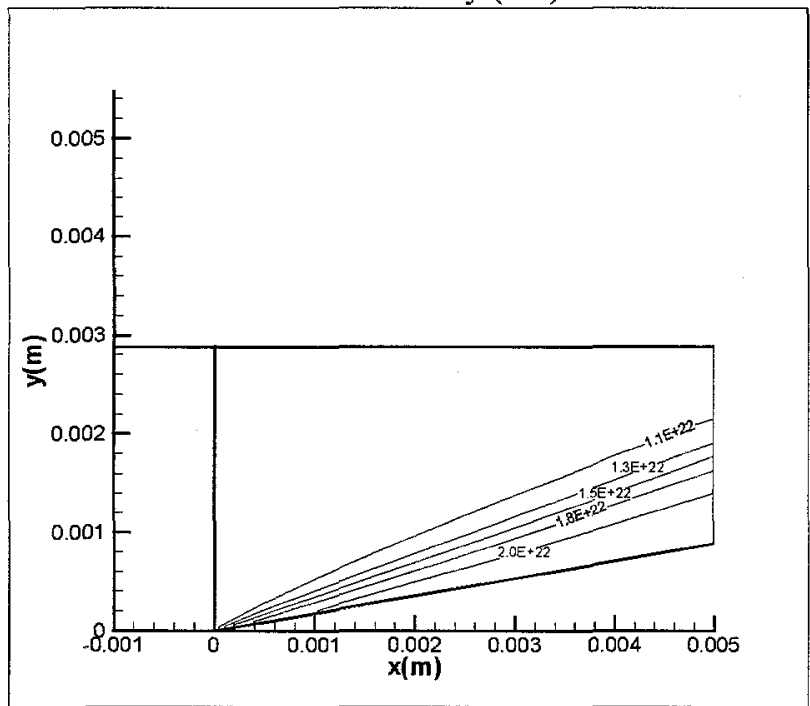

Figure 1c. Flow over a $10^{\circ}$ wedge. BGKC Simulation. Number density $\left(\mathrm{m}^{-3}\right)$

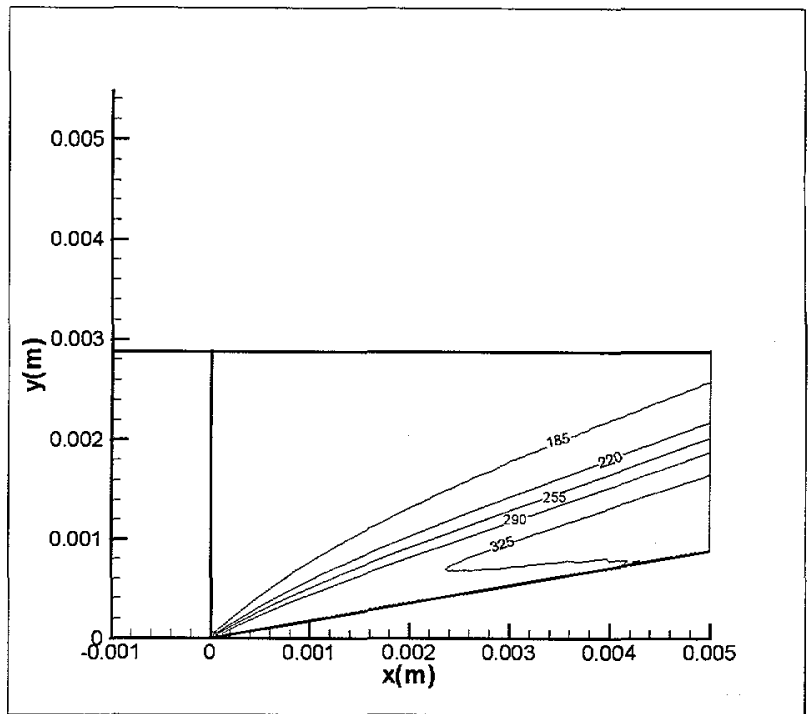

Figure 2a. Flow over a $10^{\circ}$ wedge. DSMC Simulation. Translational temperature $(\mathrm{K})$

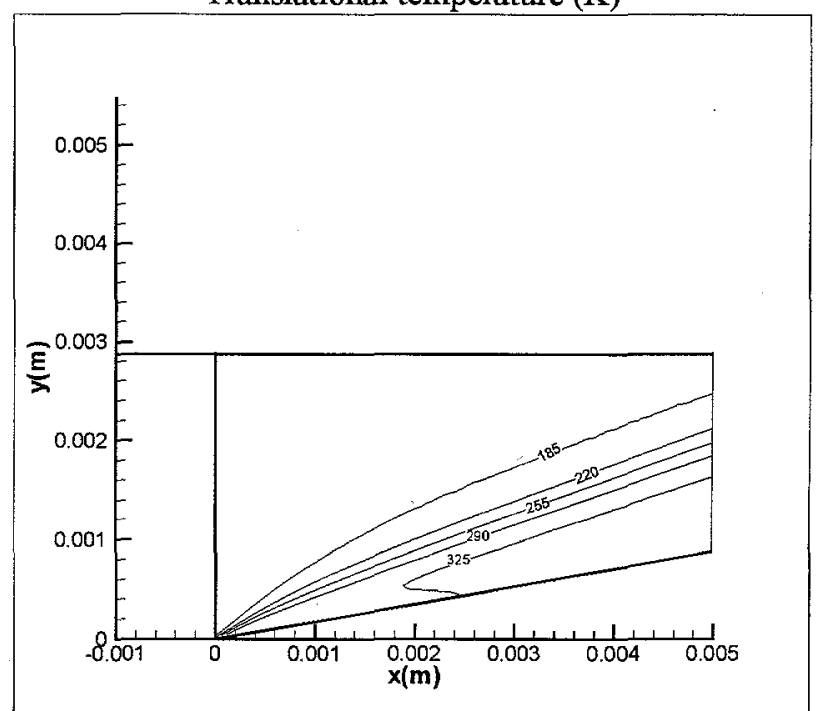

Figure 2b. Flow over a $10^{\circ}$ wedge. BGK Simulation Translational temperature $(\mathrm{K})$

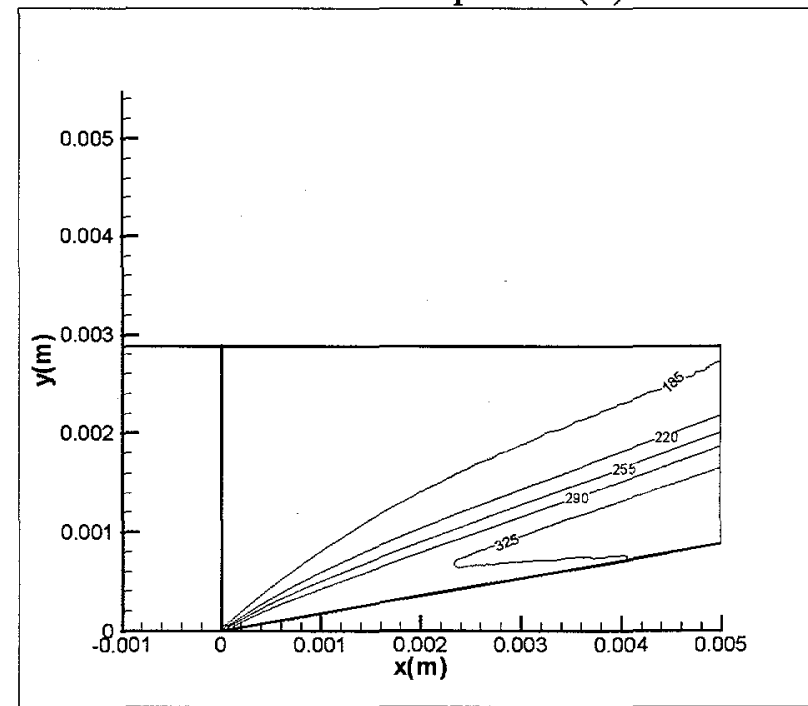

Figure 2c. Flow over a $10^{\circ}$ wedge. BGKC Simulation. Translational temperature $(\mathrm{K})$ 


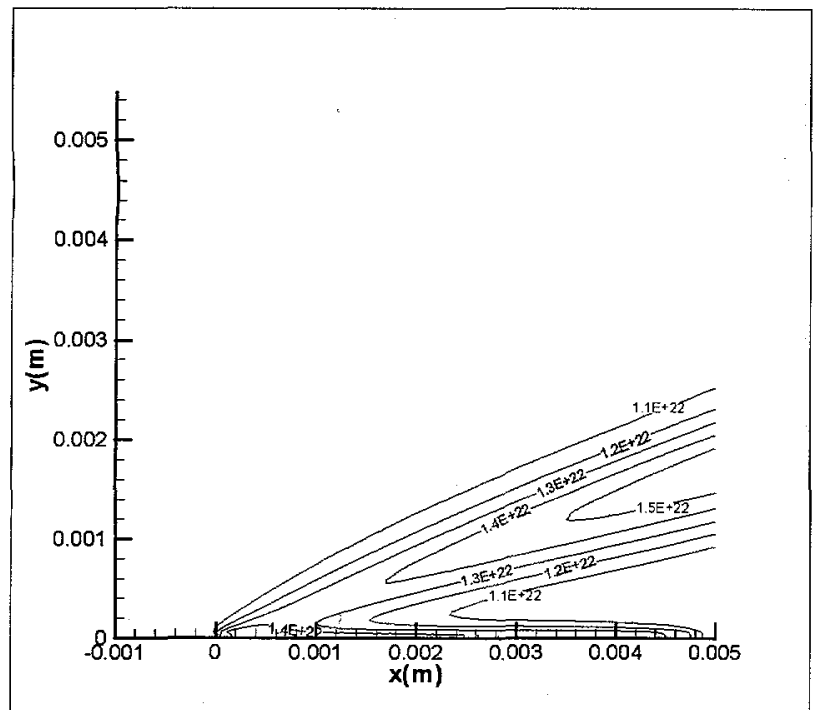

Figure 3a. Flow over a flat plate. DSMC Simulation. Number density $\left(\mathrm{m}^{-3}\right)$

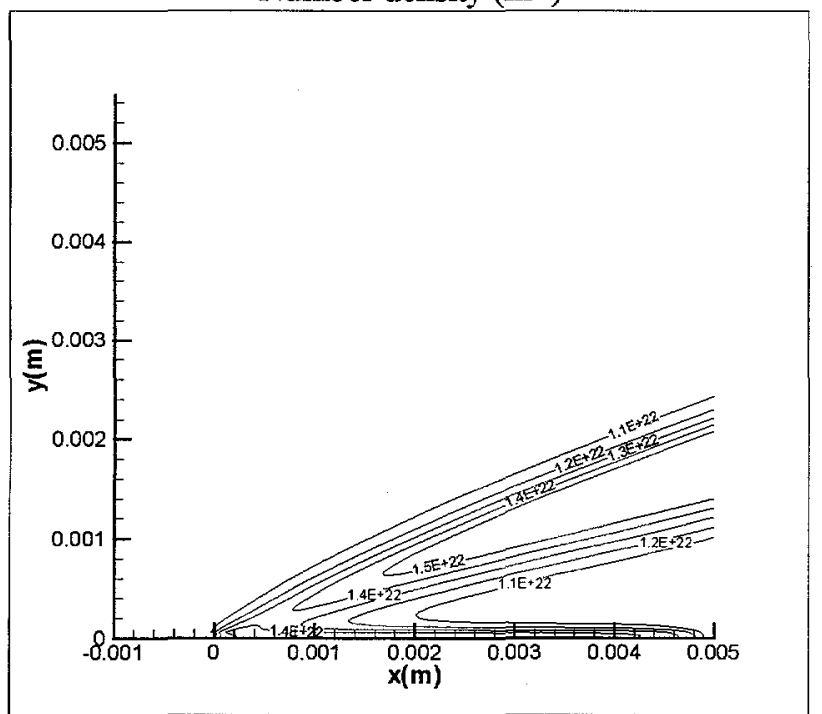

Figure 3b. Flow over a flat plate. BGK Simulation. Number density $\left(\mathrm{m}^{-3}\right)$

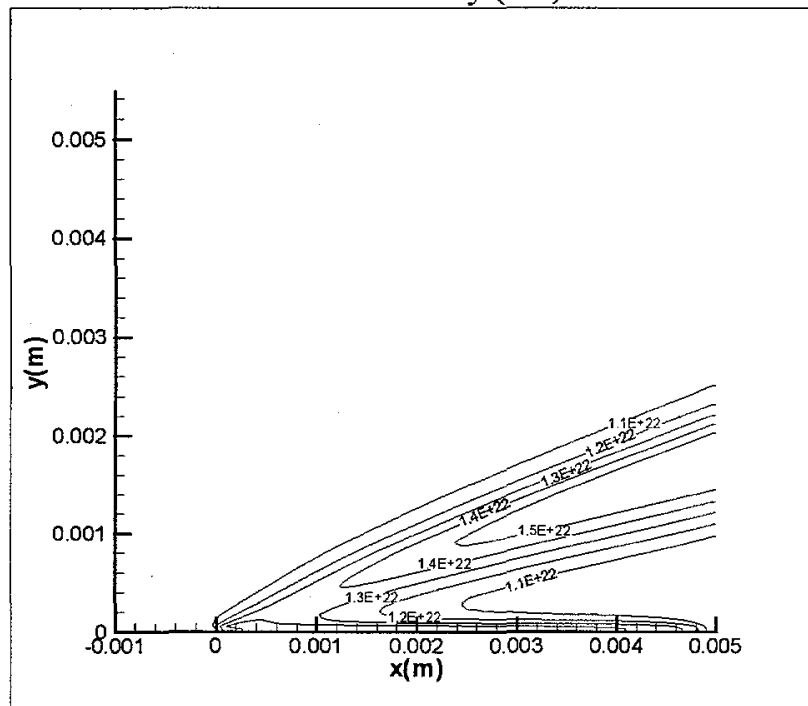

Figure 3c. Flow over a flat plate. BGKC Simulation. Number density $\left(\mathrm{m}^{-3}\right)$

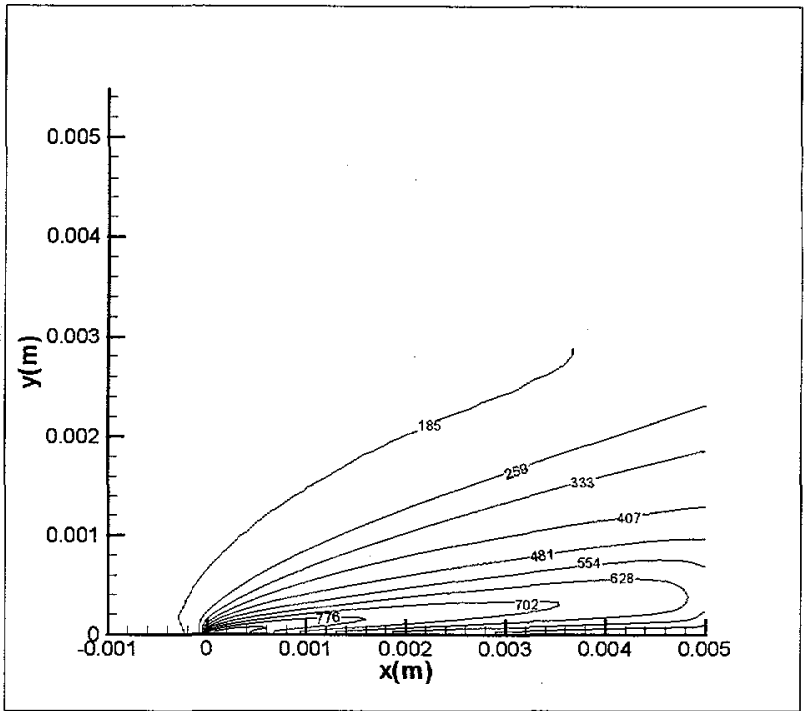

Figure 4a. Flow over a flat plate. DSMC Simulation. Translational temperature $(\mathrm{K})$

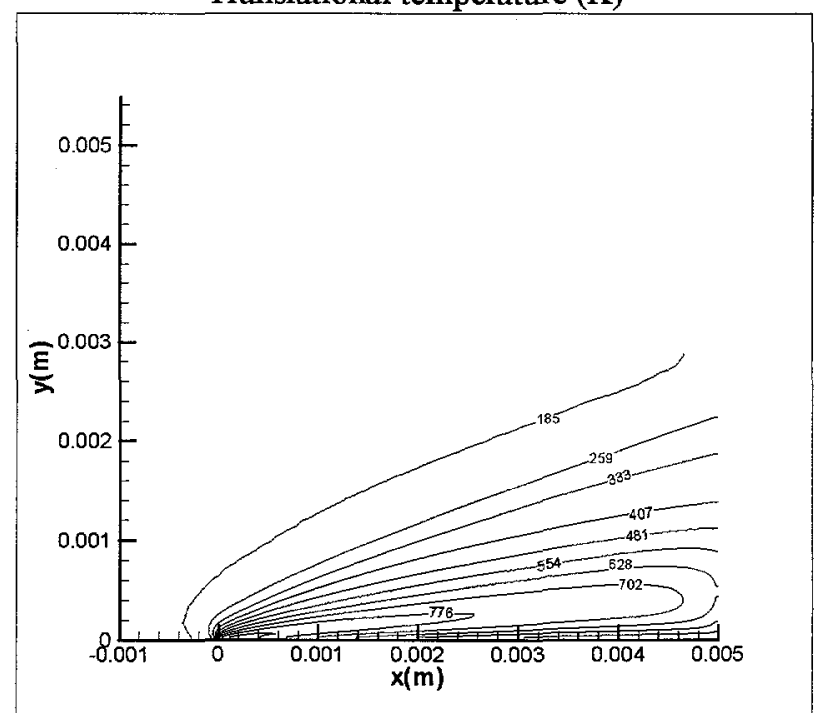

Figure 4b. Flow over a flat plate. BGK Simulation. Translational temperature (K)

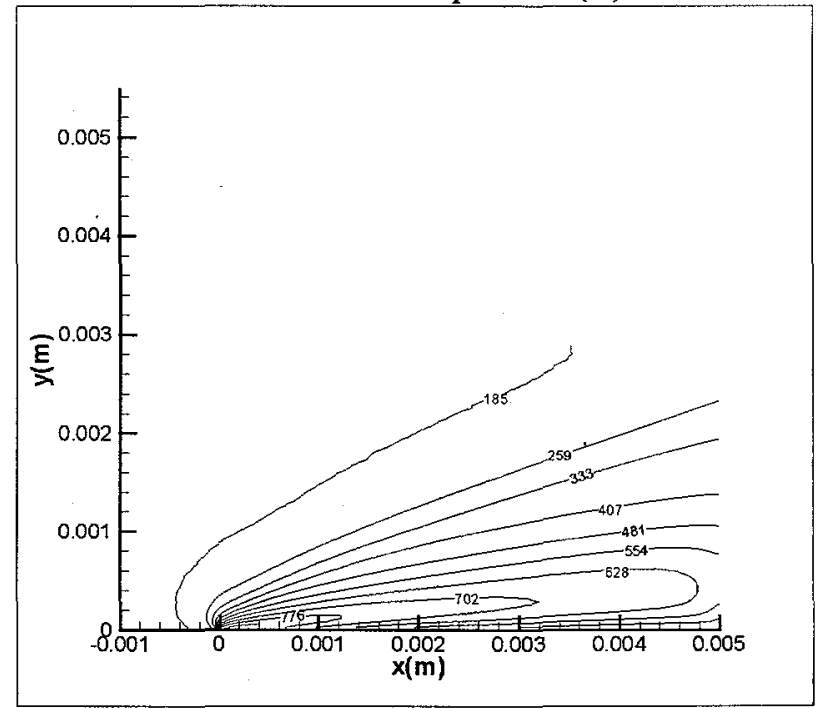

Figure 4c. Flow over a flat plate. BGKC Simulation. Translational temperature $(\mathrm{K})$ 